\title{
Boundedness of the maximal operator in the local Morrey-Lorentz spaces
}

Canay Aykol' ${ }^{1}$, Vagif S Guliyev ${ }^{2,3}$ and Ayhan Serbetci ${ }^{1 *}$

\section{${ }^{*}$ Correspondence:}

serbetci@ankara.edu.tr

'Department of Mathematics, Ankara University, Ankara, Turkey Full list of author information is available at the end of the article

\begin{abstract}
In this paper we define a new class of functions called local Morrey-Lorentz spaces $M_{p, q ; \lambda}^{\text {loc }}\left(\mathbb{R}^{n}\right), 0<p, q \leq \infty$ and $0 \leq \lambda \leq 1$. These spaces generalize Lorentz spaces such that $M_{p, q ; 0}^{\text {loc }}\left(\mathbb{R}^{n}\right)=L_{p, q}\left(\mathbb{R}^{n}\right)$. We show that in the case $\lambda<0$ or $\lambda>1$, the space $M_{p, q ; \lambda}^{\text {loc }}\left(\mathbb{R}^{n}\right)$ is trivial, and in the limiting case $\lambda=1$, the space $M_{p, q ; 1}^{\text {loc }}\left(\mathbb{R}^{n}\right)$ is the classical Lorentz space $\Lambda_{\infty, t^{\frac{1}{p}}-\frac{1}{q}}\left(\mathbb{R}^{n}\right)$. We show that for $0<q \leq p<\infty$ and $0<\lambda \leq \frac{q}{p}$, the local Morrey-Lorentz spaces $M_{p, q ; \lambda}^{\text {loc }}\left(\mathbb{R}^{n}\right)$ are equal to weak Lebesgue spaces $W L_{\frac{1}{\bar{p}}-\frac{\lambda}{q}}\left(\mathbb{R}^{n}\right)$. We get an embedding between local Morrey-Lorentz spaces and Lorentz-Morrey spaces. Furthermore, we obtain the boundedness of the maximal operator in the local Morrey-Lorentz spaces.
\end{abstract}

MSC: Primary 42B20; 42B25; 42B35; secondary 47G10

Keywords: Morrey spaces; Lorentz spaces; Lorentz-Morrey spaces; local Morrey-Lorentz spaces; maximal operator

\section{Introduction}

The aim of this paper is to define a new class of functions called local Morrey-Lorentz spaces $M_{p, q ; \lambda}^{\text {loc }}\left(\mathbb{R}^{n}\right)$ and to study the boundedness of the maximal operator in these spaces. Local Morrey-Lorentz spaces $M_{p, q ; \lambda}^{\text {loc }}\left(\mathbb{R}^{n}\right)$ are generalizations of Lorentz spaces $L_{p, q}\left(\mathbb{R}^{n}\right)$. Lorentz spaces, introduced by Lorentz in the 1950s [1, 2], are generalizations of the more familiar $L_{p}$ spaces. Lorentz spaces, which are Banach spaces, appear to be useful in the general interpolation theory by Calderón (see [3]). Peetre [4] identified Lorentz spaces as intermediate spaces for interpolation theory by Lions and Peetre (see [5]). Riviere and Sagher [6] generalized the results of Calderón contained in [3] to include Lorentz spaces having coefficients $p$ and $q$ greater than zero; similarly, Kree and Peetre generalized the results of Lions and Peetre obtained in [5].

For $x \in \mathbb{R}^{n}$ and $t>0$, let $B(x, t)$ denote the open ball centered at $x$ of radius $t$, and let $|B(x, t)|$ be the Lebesgue measure of the ball $B(x, t)$. Note that $|B(x, t)|=\omega_{n} t^{n}$, where $\omega_{n}$ denotes the volume of the unit ball in $\mathbb{R}^{n}$. Let $f$ be a locally integrable function on $\mathbb{R}^{n}$. The Hardy-Littlewood maximal function $M f$ of $f$ is defined by

$$
M f(x)=\sup _{t>0} \frac{1}{|B(x, t)|} \int_{B(x, t)}|f(y)| d y, \quad x \in \mathbb{R}^{n} .
$$

Maximal operators play an important role in the differentiability properties of functions, singular integrals and partial differential equations. They often provide a deeper and more

\section{6ringer}

(c) 2013 Aykol et al.; licensee Springer. This is an Open Access article distributed under the terms of the Creative Commons Attribution License (http://creativecommons.org/licenses/by/2.0), which permits unrestricted use, distribution, and reproduction in any medium, provided the original work is properly cited. 
simplified approach to understanding problems in these areas than other methods. It is well known that for the classical Hardy-Littlewood maximal operator the rearrangement inequality

$$
c f^{* *}(t) \leq(M f)^{*}(t) \leq C f^{* *}(t), \quad t \in(0, \infty)
$$

holds, ([7], Chapter 3, Theorem 3.8), where $f^{*}(t)$ is the non-increasing rearrangement of $f$ and

$$
f^{* *}(t)=\frac{1}{t} \int_{0}^{t} f^{*}(t) d t
$$

Mingione [8] defined the Lorentz-Morrey spaces $\mathcal{L}_{p, q ; \lambda}\left(\mathbb{R}^{n}\right)$ as follows.

Definition 1 [8] The Lorentz-Morrey spaces $\mathcal{L}_{p, q ; \lambda}\left(\mathbb{R}^{n}\right)$ is the set of all measurable functions $f$ on $\mathbb{R}^{n}$ : for $1 \leq p<\infty, 0<q<\infty$ and $0 \leq \lambda \leq n, f \in \mathcal{L}_{p, q ; \lambda}\left(\mathbb{R}^{n}\right)$ iff

$$
\|f\|_{\mathcal{L}_{p, q ; \lambda}}=\sup _{x \in \mathbb{R}^{n}, t>0} t^{-\frac{\lambda}{p}}\left\|\chi_{B(x, t)} f\right\|_{L_{p, q}}<\infty
$$

Here $\|\cdot\|_{L_{p, q}}$ denotes the Lorentz norm of a function (see preliminaries).

In [8], Section 4.1, Mingione studied the boundedness of the restricted fractional maximal operator $M_{\beta, B}$

$$
M_{\beta, B} f(x)=\sup _{B(x, t) \subset B}|B(x, t)|^{\frac{\beta}{n}-1} \int_{B(x, t)}|f(y)| d y, \quad x \in \mathbb{R}^{n},
$$

in the restricted Lorentz-Morrey spaces $\mathcal{L}_{p, q ; \lambda}(B)$, where $B$ is any ball. Mingione derived a general non-linear version, extending $a$ priori estimates and regularity results for possibly degenerate non-linear elliptic problems to the various spaces of Lorentz and LorentzMorrey type considered in [9-11].

Ragusa [12] defined the Lorentz-Morrey spaces $L_{p, q ; \lambda}\left(\mathbb{R}^{n}\right)$ and studied some embeddings between these spaces.

Definition 2 [12] The Lorentz-Morrey spaces $L_{p, q ; \lambda}\left(\mathbb{R}^{n}\right)$ is the set of all measurable functions $f$ on $\mathbb{R}^{n}$ : for $1 \leq p<\infty, 0<q<\infty$ and $0 \leq \lambda \leq n$, iff

$$
\|f\|_{L_{p, q ; \lambda}}=\sup _{x \in \mathbb{R}^{n}, t>0} t^{-\frac{\lambda}{q}}\left\|\chi_{B(x, t)} f\right\|_{L_{p, q}}<\infty .
$$

Accordingly, $f$ belongs to

$$
L_{p, \infty ; \lambda}\left(\mathbb{R}^{n}\right) \equiv W L_{p, \lambda}\left(\mathbb{R}^{n}\right) \quad \text { iff } \quad\|f\|_{L_{p, \infty ; \lambda}}=\|f\|_{W L_{p, \lambda}}<\infty
$$

Note that the spaces $\mathcal{L}_{p, q ; \lambda}\left(\mathbb{R}^{n}\right)$ and $L_{p, q ; \lambda}\left(\mathbb{R}^{n}\right)$ defined by Mingione and Ragusa respectively coincide, thus

$$
\mathcal{L}_{p, q ; \lambda}\left(\mathbb{R}^{n}\right)=L_{p, q ; \lambda} \frac{q}{p}\left(\mathbb{R}^{n}\right)
$$


Recall that the local Morrey-type spaces $L M_{p \theta, w}$ were introduced and the boundedness in these spaces of the fractional integral operators and singular integral operators defined on homogeneous Lie groups were proved by Guliyev [13] in the doctoral thesis (see, also [14-16]). They are given by

$$
\|f\|_{L M_{p \theta, w}}=\|w(r)\| f\left\|_{L_{p}(B(0, r))}\right\|_{L_{\theta}(0, \infty)}
$$

where $w$ is a positive measurable function defined on $(0, \infty)$. Some necessary and sufficient conditions for the boundedness of the maximal, fractional maximal, Riesz potential and singular integral operators in the local Morrey-type space $L M_{p \theta, w}$ were given in [17-21]. We should explain that the spaces $L M_{p \theta, w}$ are closely related to the $B_{\sigma}$ spaces (see [22,23]).

This paper is organized as follows. In Section 2 we give some notations and definitions of the Morrey, Lorentz and classical Lorentz spaces. In Section 3 we define a new class of functions called local Morrey-Lorentz spaces $M_{p, q ; \lambda}^{\text {loc }}\left(\mathbb{R}^{n}\right), 0<p, q \leq \infty$ and $0 \leq \lambda \leq 1$. These spaces generalize Lorentz spaces such that $M_{p, q ; 0}^{\text {loc }}\left(\mathbb{R}^{n}\right)=L_{p, q}\left(\mathbb{R}^{n}\right)$. We show that in the case $\lambda<0$ or $\lambda>1$, the space $M_{p, q ; \lambda}^{\text {loc }}\left(\mathbb{R}^{n}\right)$ is trivial, and in the limiting case $\lambda=1$, the space

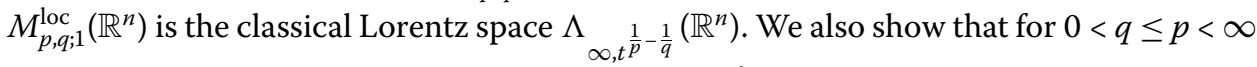
and $0<\lambda \leq \frac{q}{p}$, the local Morrey-Lorentz spaces $M_{p, q ; \lambda}^{\text {loc }}\left(\mathbb{R}^{n}\right)$ are equal to weak Lebesgue spaces $W L_{\frac{1}{p}-\frac{\lambda}{q}}\left(\mathbb{R}^{n}\right)$. In Section 4 we prove the boundedness of the maximal operator in $M_{p, q ; \lambda}^{\mathrm{loc}}\left(\mathbb{R}^{n}\right)$.

Throughout the paper, we write $A \lesssim B$ if there exists a positive constant $C$, independent of appropriate quantities such as functions, satisfying $A \leq C B$. If $p \in[1, \infty]$, the conjugate number $p^{\prime}$ is defined by $p^{\prime}=\frac{p}{p-1}$ and if $p \in(0,1)$, the conjugate number $p^{\prime}$ is defined by $p^{\prime}=\frac{p}{1-p}$.

\section{Preliminaries}

Let $E$ be a measurable subset of $\mathbb{R}^{n}$ and $|E|=\int_{E} d x$. We denote by $L_{p}(E)$ the class of all measurable functions $f$ defined on $E$ for which

$$
\begin{aligned}
& \|f\|_{L_{p}(E)}:=\left(\int_{E}|f(y)|^{p} d y\right)^{\frac{1}{p}}<\infty, \quad 0<p<\infty, \\
& \|f\|_{L_{\infty}(E)}:=\sup \{\alpha:|\{y \in E:|f(y)| \geq \alpha\}|>0\} .
\end{aligned}
$$

We define rearrangement of $f$ in decreasing order by

$$
f^{*}(t)=\inf \left\{\lambda>0: \mu_{f}(\lambda) \leq t\right\}, \quad \forall t \in(0, \infty)
$$

where $\mu_{f}(\lambda)$ denotes the distribution function of $f$ given by

$$
\mu_{f}(\lambda)=\left|\left\{y \in \mathbb{R}^{n}:|f(y)|>\lambda\right\}\right| .
$$

We denote by $W L_{p}\left(\mathbb{R}^{n}\right)$ the weak $L_{p}$ space of all measurable functions $f$ with the quasinorm

$$
\|f\|_{W L_{p}}=\sup _{t>0} t^{1 / p} f^{*}(t), \quad 1 \leq p<\infty
$$


Now we recall the definitions of Morrey spaces, Lorentz spaces and classical Lorentz spaces.

Morrey spaces were introduced by Morrey [24] in 1938 in connection with certain problems in elliptic partial differential equations and calculus of variations. Later, Morrey spaces found important applications to Navier-Stokes and Schrödinger equations, elliptic problems with discontinuous coefficients, and potential theory.

Definition $3[24,25]$ We denote by $L_{p, \lambda}\left(\mathbb{R}^{n}\right)$ the Morrey space for $0 \leq \lambda \leq n, 1 \leq p<\infty$, $f \in L_{p, \lambda}\left(\mathbb{R}^{n}\right)$ if $f \in L_{p}^{\text {loc }}\left(\mathbb{R}^{n}\right)$ and

$$
\|f\|_{L_{p, \lambda}}=\sup _{x \in \mathbb{R}^{n}, t>0} t^{-\frac{\lambda}{p}}\|f\|_{L_{p(B(x, t)}}<\infty
$$

If $\lambda=0$, then $L_{p, 0}\left(\mathbb{R}^{n}\right)=L_{p}\left(\mathbb{R}^{n}\right)$; if $\lambda=n$, then $L_{p, n}\left(\mathbb{R}^{n}\right)=L_{\infty}\left(\mathbb{R}^{n}\right)$; if $\lambda<0$ or $\lambda>n$, then $L_{p, \lambda}\left(\mathbb{R}^{n}\right)=\Theta$, where $\Theta$ is the set of all functions equivalent to 0 on $\mathbb{R}^{n}$.

Also, by $W L_{p, \lambda}\left(\mathbb{R}^{n}\right)$ we denote the weak Morrey space of all functions $f \in W L_{p}^{\text {loc }}\left(\mathbb{R}^{n}\right)$ for which

$$
\|f\|_{W L_{p, \lambda}}=\sup _{x \in \mathbb{R}^{n}, t>0} t^{-\frac{\lambda}{p}}\|f\|_{W L_{p(B(x, t))}}<\infty .
$$

Lorentz spaces were introduced by Lorentz in 1950. Lorentz spaces, which are Banach spaces and generalizations of the more familiar $L_{p}$ spaces, appear to be useful in the general interpolation theory.

Definition 4 The Lorentz space $L_{p, q}\left(\mathbb{R}^{n}\right), 0<p, q \leq \infty$, is the collection of all measurable functions $f$ on $\mathbb{R}^{n}$ such the quantity

$$
\|f\|_{L_{p, q}}=\left\|t^{\frac{1}{\bar{p}}-\frac{1}{q}} f^{*}(t)\right\|_{L_{q}(0, \infty)}
$$

is finite.

Note that $L_{p, \infty}\left(\mathbb{R}^{n}\right)=W L_{p}\left(\mathbb{R}^{n}\right)$ (see, for example, [26]).

If $p=q=\infty$, then the space $L_{\infty, \infty}$ is denoted by $L_{\infty}$.

If $1 \leq q \leq p$ or $p=q=\infty$, then the functional $\|f\|_{p, q}$ is a norm.

For $0<q \leq p \leq r \leq \infty$, we have, with continuous embeddings, that

$$
L_{p, q} \subset L_{p} \subset L_{p, r} \subset W L_{p} .
$$

The function $f^{* *}:(0, \infty) \rightarrow[0, \infty]$ is defined as

$$
f^{* *}(t)=\frac{1}{t} \int_{0}^{t} f^{*}(s) d s
$$

In the case $0<p, q \leq \infty$, we give a functional $\|\cdot\|_{L p, q}^{*}$ by

$$
\|f\|_{L_{p, q}}^{*}:=\|f\|_{L_{p, q}(0, \infty)}^{*}=\left\|t^{\frac{1}{p}-\frac{1}{q}} f^{* *}(t)\right\|_{L_{q}(0, \infty)}
$$


(with the usual modification if $0<p \leq \infty, q=\infty$ ), which is a norm on $L_{p, q}\left(\mathbb{R}^{n}\right)$ for $1<p<$ $\infty, 1 \leq q \leq \infty$ or $p=q=\infty$.

If $1<p \leq \infty, 1 \leq q \leq \infty$, then for $1 \leq r<p$ (see [27]),

$$
\|f\|_{L_{p, q}} \leq\|f\|_{L_{p, q}}^{*} \leq\left(\frac{p}{p-r}\right)^{1 / r}\|f\|_{L_{p, q}} .
$$

We denote by $\mathfrak{M}\left(\mathbb{R}^{n}\right)$ the set of all extended real-valued measurable functions on $\mathbb{R}^{n}$ and by $\mathfrak{M}^{+}(0, \infty)$ the set of all non-negative measurable functions on $(0, \infty)$.

Definition 5 Let $0<p \leq \infty$ and $\psi \in \mathfrak{M}^{+}(0, \infty)$. We denote by $\Lambda_{p, \psi}\left(\mathbb{R}^{n}\right)$ the classical Lorentz spaces, the spaces of all measurable functions with a finite quasi-norm

$$
\Lambda_{p, \psi}\left(\mathbb{R}^{n}\right):=\left\{f \in \mathfrak{M}\left(\mathbb{R}^{n}\right):\|f\|_{\Lambda_{p, \psi}}:=\left\|\psi f^{*}\right\|_{L_{p}(0, \infty)}\right\}
$$

Therefore, for $\psi(t)=t^{\frac{1}{p}-\frac{1}{q}}, 0<p, q \leq \infty$, we get $\Lambda_{p, t \frac{1}{p}-\frac{1}{q}}\left(\mathbb{R}^{n}\right)=L_{p, q}\left(\mathbb{R}^{n}\right)$ with equality of 'norms'.

Useful references for Lorentz spaces are, for instance, in [7, 28, 29].

Remark 1 Since $\|f\|_{L_{p, p}}=\|f\|_{L_{p}}$, it can be easily shown that $L_{p, p ; \lambda} \equiv L_{p, \lambda}$.

\section{Local Morrey-Lorentz spaces $M_{p, q ; \lambda}^{\text {loc }}\left(\mathbb{R}^{n}\right)$}

In this section we define the local Morrey-Lorentz spaces $M_{p, q ; \lambda}^{\text {loc }}\left(\mathbb{R}^{n}\right), 0<p, q \leq \infty$ and $0 \leq \lambda \leq 1$. These spaces generalize Lorentz spaces so that $M_{p, q ; 0}^{\text {loc }}\left(\mathbb{R}^{n}\right)=L_{p, q}\left(\mathbb{R}^{n}\right)$. We show that in the case $\lambda<0$ or $\lambda>1$, the space $M_{p, q ; \lambda}^{\text {loc }}\left(\mathbb{R}^{n}\right)$ is trivial, and in the limiting case $\lambda=1$, the space $M_{p, q ; 1}^{\text {loc }}\left(\mathbb{R}^{n}\right)$ is the classical Lorentz space $\Lambda_{\infty, t^{\frac{1}{p}}-\frac{1}{q}}\left(\mathbb{R}^{n}\right)$. We also show that for $0<q \leq p<\infty$ and $0<\lambda \leq \frac{q}{p}$, the local Morrey-Lorentz spaces $M_{p, q ; \lambda}^{\text {loc }}\left(\mathbb{R}^{n}\right)$ are equal to weak Lebesgue spaces $W L_{\frac{1}{p}-\frac{\lambda}{q}}\left(\mathbb{R}^{n}\right)$.

Definition 6 Let $0<p, q \leq \infty$ and let $0 \leq \lambda \leq 1$. We denote by $M_{p, q ; \lambda}^{\text {loc }}\left(\mathbb{R}^{n}\right)$ the local Morrey-Lorentz spaces, the spaces of all measurable functions with a finite quasi-norm

$$
\|f\|_{M_{p, q ; \lambda}^{\text {loc }}}:=\sup _{t>0} t^{-\frac{\lambda}{q}}\left\|s^{\frac{1}{p}-\frac{1}{q}} f^{*}(s)\right\|_{L_{q}(0, t)}
$$

If $\lambda<0$ or $\lambda>1$, then $M_{p, q ; \lambda}^{\text {loc }}\left(\mathbb{R}^{n}\right)=\Theta$, where $\Theta$ is the set of all functions equivalent to 0 on $\mathbb{R}^{n}$. Also, $M_{p, q ; 0}^{\text {loc }}\left(\mathbb{R}^{n}\right)=L_{p, q}\left(\mathbb{R}^{n}\right)$. In the case $q=p$, we denote the space $M_{p, q ; \lambda}^{\text {loc }}\left(\mathbb{R}^{n}\right)$ by $M_{p, \lambda}^{\mathrm{loc}}\left(\mathbb{R}^{n}\right)$.

Lemma 1 Let $0<p, q \leq \infty$. Then

$$
M_{p, q ; 1}^{\mathrm{loc}}\left(\mathbb{R}^{n}\right)=\Lambda_{\infty, t} \frac{1}{p-\frac{1}{q}}\left(\mathbb{R}^{n}\right)
$$

and

$$
\|f\|_{M_{p, q ; 1}^{\mathrm{loc}}}=\|f\|_{\Lambda}{ }_{\infty, t}^{\frac{1}{p}-\frac{1}{q}} \equiv\left\|\tau^{\frac{1}{p}-\frac{1}{q}} f^{*}(\tau)\right\|_{L_{\infty}(0, \infty)} .
$$


Proof Let $f \in \Lambda_{\infty, t^{\frac{1}{p}-\frac{1}{q}}}\left(\mathbb{R}^{n}\right)$. Then

$$
\left(t^{-1} \int_{0}^{t} \tau^{\frac{q}{p}-1} f^{*}(\tau)^{q} d \tau\right)^{1 / q} \leq\|f\|_{\Lambda, t^{\frac{1}{p}-\frac{1}{q}}}
$$

Therefore $f \in M_{p, q ; 1}^{\text {loc }}\left(\mathbb{R}^{n}\right)$ and

$$
\|f\|_{M_{p, q ; 1}^{\mathrm{loc}}} \leq\|f\|_{\Lambda} \underset{\infty, t^{\frac{1}{p}-\frac{1}{q}}}{ } .
$$

Let $f \in M_{p, q ; 1}^{\text {loc }}\left(\mathbb{R}^{n}\right)$. By the Lebesgue theorem, we have

$$
\lim _{s \rightarrow 0} \frac{1}{2 s} \int_{t-s}^{t+s} \tau^{\frac{q}{p}-1} f^{*}(\tau)^{q} d \tau=t^{\frac{q}{p}-1} f^{*}(t)^{q}
$$

Then

$$
\begin{aligned}
t^{\frac{1}{p}-\frac{1}{q}} f^{*}(t) & =\left(\lim _{s \rightarrow 0} \frac{1}{2 s} \int_{t-s}^{t+s} \tau^{\frac{q}{p}-1} f^{*}(\tau)^{q} d \tau\right)^{1 / q} \\
& \leq 2^{-\frac{1}{q}} \sup _{0<s<t} s^{-\frac{1}{q}}\left\|\tau^{\frac{1}{p}-\frac{1}{q}} f^{*}(\tau)\right\|_{L_{q}(t-s, t+s)} \\
& =\left\|\tau^{\frac{1}{p}-\frac{1}{q}} f^{*}(\tau)\right\|_{L_{\infty}(0, \infty)} \\
& =\|f\|_{M_{p, q ; 1}^{\mathrm{loc}} .} .
\end{aligned}
$$

Therefore $f \in \Lambda_{\infty, t^{\frac{1}{p}} \frac{1}{q}}\left(\mathbb{R}^{n}\right)$ and

$$
\|f\|_{\Lambda, t \frac{1}{p}-\frac{1}{q}} \leq\|f\|_{M_{p, q ; 1}^{\mathrm{loc}}} .
$$

Corollary 1 Let $0<p \leq \infty$. Then

$$
M_{p, 1}^{\text {loc }}\left(\mathbb{R}^{n}\right)=L_{\infty}\left(\mathbb{R}^{n}\right)
$$

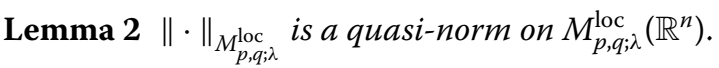

Proof From the definition $f^{*} \geq 0$. This implies that $\|f\|_{M_{p, q ; \lambda}^{\text {loc }}} \geq 0$. Moreover, $\|f\|_{M_{p, q ; \lambda}^{\text {loc }}}=0$ implies that $\left\|s^{\frac{1}{p}-\frac{1}{q}} f^{*}(s)\right\|_{L_{q}(0, t)}=0$ for all $t>0$. Hence, we get $f^{*}=0$ a.e. Thus, $f=0$ since $f$ is representative of an equivalence class.

Now, let $a \neq 0$ be a real constant, $f \in M_{p, q ; \lambda}^{\mathrm{loc}}\left(\mathbb{R}^{n}\right)$. Noting

$$
(a f)^{*}(t)=|a| f^{*}(t), \quad t>0,
$$

the homogeneity condition follows

$$
\|a f\|_{M_{p, q ; \lambda}^{\mathrm{loc}}}=|a|\|f\|_{M_{p, q ; \lambda}^{\mathrm{loc}}}
$$


Let $f, g \in M_{p, q ; \lambda}^{\text {loc }}\left(\mathbb{R}^{n}\right)$ and $0<p, q<\infty$. Since $(f+g)^{*}(t) \leq f^{*}\left(\frac{t}{2}\right)+g^{*}\left(\frac{t}{2}\right)$, for any $t>0$, we have

$$
\begin{aligned}
\int_{0}^{t}\left((f+g)^{*}(s) s^{1 / p}\right)^{q} \frac{d s}{s} & \leq \max \left\{1,2^{q-1}\right\}\left(\int_{0}^{t}\left(f^{*}\left(\frac{s}{2}\right) s^{1 / p}\right)^{q} \frac{d s}{s}+\int_{0}^{t}\left(g^{*}\left(\frac{s}{2}\right) s^{1 / p}\right)^{q} \frac{d s}{s}\right) \\
& \leq 2^{\frac{q}{p}} \max \left\{1,2^{q-1}\right\}\left(\int_{0}^{\frac{t}{2}}\left(f^{*}(s) s^{1 / p}\right)^{q} \frac{d s}{s}+\int_{0}^{\frac{t}{2}}\left(g^{*}(s) s^{1 / p}\right)^{q} \frac{d s}{s}\right) .
\end{aligned}
$$

Therefore we get

$$
\|f+g\|_{M_{p, q ; \lambda}^{\mathrm{loc}}} \leq 2^{\frac{1}{p}} \max \left\{1,2^{1-\frac{1}{q}}\right\}\left(\|f\|_{M_{p, q ; \lambda}^{\mathrm{loc} ;}}+\|g\|_{M_{p, q ; \lambda}^{\mathrm{loc}}}\right) .
$$

Let $f, g \in M_{p, \infty ; \lambda}^{\text {loc }}\left(\mathbb{R}^{n}\right), 0<p \leq \infty$ and $q=\infty$. Then

$$
\begin{aligned}
\sup _{t>0} t^{1 / p}(f+g)^{*}(t) & \leq \sup _{t>0} t^{1 / p}\left(f^{*}\left(\frac{t}{2}\right)+g^{*}\left(\frac{t}{2}\right)\right) \\
& =\sup _{u>0}(2 u)^{1 / p}\left(f^{*}(u)+g^{*}(u)\right) \\
& \leq 2^{1 / p}\left(\sup _{u>0} u^{1 / p} f^{*}(u)+\sup _{u>0} u^{1 / p} g^{*}(u)\right) \\
& =2^{1 / p}\left(\|f\|_{L_{p, \infty}}+\|g\|_{L_{p, \infty}}\right) .
\end{aligned}
$$

Therefore we get

$$
\|f+g\|_{M_{p, \infty ; \lambda}^{\mathrm{loc}}} \leq 2^{\frac{1}{p}}\left(\|f\|_{M_{p, \infty ; \lambda}^{\mathrm{loc}}}+\|g\|_{M_{p, \infty ; \lambda}^{\mathrm{loc}}}\right) .
$$

The following theorem states that $M_{p, q ; \lambda}^{\text {loc }}\left(\mathbb{R}^{n}\right)$ local Morrey-Lorentz spaces are equivalent to $W L_{q}\left(\mathbb{R}^{n}\right)$ weak Lebesgue spaces in the case $\frac{1}{q}=\frac{1}{p}-\frac{\lambda}{q}$ and $0<\lambda \leq \frac{q}{p}$.

Theorem 1 Let $0<q \leq p<\infty, \frac{1}{r}=\frac{1}{p}-\frac{\lambda}{q}$ and $0<\lambda \leq \frac{q}{p}$. Then

$$
M_{p, q ; \lambda}^{\mathrm{loc}}\left(\mathbb{R}^{n}\right) \equiv W L_{r}\left(\mathbb{R}^{n}\right)
$$

and

$$
\left(\frac{q}{p}\right)^{-\frac{1}{q}}\|f\|_{W L_{r}} \leq\|f\|_{M_{p, q ; \lambda}^{\mathrm{loc}}} \leq \lambda^{-\frac{1}{q}}\|f\|_{W L_{r}}, \quad f \in W L_{r}\left(\mathbb{R}^{n}\right)
$$

Proof Suppose $f \in M_{p, q ; \lambda}^{\mathrm{loc}}\left(\mathbb{R}^{n}\right)$. Then, from the monotonicity of $f^{*}$ on $(0, \infty)$ for all $t>0$, we get

$$
\begin{aligned}
t^{\frac{1}{r}} f^{*}(t) & =t^{-\frac{\lambda}{q}+\frac{1}{p}} f^{*}(t) \\
& =\left(\frac{q}{p}\right)^{\frac{1}{q}}\left(t^{-\lambda} f^{*}(t)^{q} \int_{0}^{t} s^{\frac{q}{p}-1} d s\right)^{\frac{1}{q}} \\
& \leq\left(\frac{q}{p}\right)^{\frac{1}{q}}\left(t^{-\lambda} \int_{0}^{t}\left(f^{*}(s) s^{\frac{1}{p}}\right)^{q} \frac{d s}{s}\right)^{\frac{1}{q}} \leq\left(\frac{q}{p}\right)^{\frac{1}{q}}\|f\|_{M_{p, q ;}^{\mathrm{loc}},} .
\end{aligned}
$$


Thus, $f \in W L_{r}\left(\mathbb{R}^{n}\right)$ and

$$
\|f\|_{W L_{r}}:=\sup _{t>0} t^{\frac{1}{r}} f^{*}(t) \leq\left(\frac{q}{p}\right)^{\frac{1}{q}}\|f\|_{M_{p, q ; \lambda}^{\mathrm{loc}}} .
$$

Let $f \in W L_{r}\left(\mathbb{R}^{n}\right)$, where $\frac{1}{r}=\frac{1}{p}-\frac{\lambda}{q}$. Then, for all $t>0$,

$$
t^{\frac{1}{r}} f^{*}(t)=t^{-\frac{\lambda}{q}+\frac{1}{p}} f^{*}(t) \leq\|f\|_{W L_{r}} .
$$

This implies that

$$
f^{*}(t) \leq\|f\|_{W L_{r}} t^{\frac{\lambda}{q}-\frac{1}{p}}, \quad t>0 .
$$

Therefore we get

$$
\left(f^{*}(t) t^{\frac{1}{p}}\right)^{q} \leq\|f\|_{W L_{r}} t^{\lambda}, \quad t>0 .
$$

Consequently,

$$
\begin{aligned}
\|f\|_{M_{p, q ; \lambda}^{\mathrm{loc}}} & =\sup _{t>0}\left(t^{-\lambda} \int_{0}^{t}\left(f^{*}(s) s^{\frac{1}{p}}\right)^{q} \frac{d s}{s}\right)^{\frac{1}{q}} \\
& \leq\|f\|_{W L_{r}} \sup _{t>0}\left(t^{-\lambda} \int_{0}^{t} s^{\lambda-1} d s\right)^{\frac{1}{q}}=\lambda^{-\frac{1}{q}}\|f\|_{W L_{r} .}
\end{aligned}
$$

Corollary 2 Let $0<q \leq p<\infty$. Then

$$
M_{p, q ; \frac{q}{p}}^{\mathrm{loc}}\left(\mathbb{R}^{n}\right) \equiv L_{\infty}\left(\mathbb{R}^{n}\right)
$$

The following embedding is valid.

Lemma 3 Let $0<p, q<\infty$ and $0 \leq \lambda \leq 1$. Then

$$
M_{p, q ; \lambda}^{\mathrm{loc}}\left(\mathbb{R}^{n}\right) \hookrightarrow L_{p, q ; n \lambda}\left(\mathbb{R}^{n}\right)
$$

and for $f \in M_{p, q ; \lambda}^{\mathrm{loc}}\left(\mathbb{R}^{n}\right)$

$$
\|f\|_{L_{p, q ; n \lambda}} \lesssim\|f\|_{M_{p, q ; \lambda}^{\mathrm{loc}}}
$$

Proof For $x \in \mathbb{R}^{n}$ and $t>0$, from the monotonicity of $f^{*}$ on $(0, \infty)$, we get

$$
\begin{aligned}
\left\|f \chi_{B(x, t)}\right\|_{L_{p, q}} & =\left(\int_{0}^{\infty} s^{\frac{q}{p}-1}\left(\left(f \chi_{B(x, t)}\right)^{*}(s)\right)^{q} d s\right)^{1 / q} \\
& \leq\left(\int_{0}^{\infty} s^{\frac{q}{p}-1}\left(\left(f \chi_{B(x, t)}\right)^{* *}(s)\right)^{q} d s\right)^{1 / q} \\
& =\left(\int_{0}^{\infty} s^{\frac{q}{p}-1}\left(\frac{1}{s} \int_{0}^{s}\left(f \chi_{B(x, t)}\right)^{*}(\tau) d \tau\right)^{q} d s\right)^{1 / q} .
\end{aligned}
$$


From the inequality $(f g)^{*}(s) \leq f^{*}(s) g^{*}(s)$ and the equality $\left(\chi_{B}\right)^{*}(s)=\chi_{[0,|B|)}(s)$, we get

$$
\left(f \chi_{B(x, t)}\right)^{*}(s) \leq f^{*}(s) \chi_{\left[0, \omega_{n} t^{n}\right)}(s)
$$

For $x \in \mathbb{R}^{n}$ and $t>0$, we have

$$
\begin{aligned}
\| f & \chi_{B(x, t)} \|_{L_{p, q}} \\
& \leq\left(\int_{0}^{\infty} \tau^{\frac{q}{p}-1}\left(\frac{1}{\tau} \int_{0}^{\tau} f^{*}(s) \chi_{\left[0, \omega_{n} t^{n}\right)}(s) d s\right)^{q} d \tau\right)^{1 / q} \\
& \lesssim\left(\int_{0}^{\omega_{n} t^{n}} \tau^{\frac{q}{p}-1}\left(\frac{1}{\tau} \int_{0}^{\tau} f^{*}(s) d s\right)^{q} d \tau\right)^{1 / q}+\int_{0}^{\omega_{n} t^{n}} f^{*}(s) d s\left(\int_{\omega_{n} t^{n}}^{\infty} \tau^{\frac{q}{p}-1-q} d \tau\right)^{1 / q} \\
& =\left(\int_{0}^{\omega_{n} t^{n}} \tau^{\frac{q}{p}-1}\left(\frac{1}{\tau} \int_{0}^{\tau} f^{*}(s) d s\right)^{q} d \tau\right)^{1 / q}+\left(\frac{p^{\prime}}{q}\right)^{\frac{1}{q}} \omega_{n}^{-\frac{1}{p^{\prime}}} t^{-\frac{n}{p^{\prime}}}\left(\int_{0}^{\omega_{n} t^{n}} f^{*}(s) d s\right)^{1 / q} .
\end{aligned}
$$

From the Hölder inequality we obtain

$$
\begin{aligned}
\int_{0}^{\omega_{n} t^{n}} f^{*}(s) d s & \leq\left(\int_{0}^{\omega_{n} t^{n}} f^{*}(s)^{q} s^{\frac{q}{p}-1} d s\right)^{1 / q}\left(\int_{0}^{\omega_{n} t^{n}} s^{\left(\frac{1}{q}-\frac{1}{p}\right) q^{\prime}} d s\right)^{1 / q^{\prime}} \\
& =\left(\left(\frac{1}{q}-\frac{1}{p}\right) q^{\prime}+1\right)^{-\frac{1}{q^{\prime}}}\left(\omega_{n} t^{n}\right)^{\frac{1}{p^{\prime}}}\left(\int_{0}^{\omega_{n} t^{n}} f^{*}(s)^{q} s^{\frac{q}{p}-1} d s\right)^{1 / q} .
\end{aligned}
$$

Then

$$
\left(\int_{0}^{\infty} s^{\frac{q}{p}-1}\left(\frac{1}{s} \int_{0}^{\min \left\{s, \omega_{n} t^{n}\right\}} f^{*}(\tau) d \tau\right)^{q} d s\right)^{1 / q} \lesssim\left(\int_{0}^{\omega_{n} t^{n}} s^{\frac{q}{p}-1} f^{*}(s)^{q} d s\right)^{1 / q}
$$

Therefore

$$
\left\|f \chi_{B(x, t)}\right\|_{L_{p, q}} \lesssim\left\|s^{\frac{1}{\bar{p}}-\frac{1}{q}} f^{*}(s)\right\|_{L_{q}\left(0, \omega_{n} t^{n}\right.} .
$$

Consequently,

$$
\begin{aligned}
\|f\|_{L_{p, q ; n \lambda}} & =\sup _{x \in \mathbb{R}^{n}} \sup _{t>0} t^{-\frac{n \lambda}{q}}\left\|f \chi_{B(x, t)}\right\|_{L_{p, q}} \\
& \lesssim \sup _{t>0} t^{-\frac{n \lambda}{q}}\left\|s^{\frac{1}{p}-\frac{1}{q}} f^{*}(s)\right\|_{L_{q}\left(0, \omega_{n} t^{n}\right)} \\
& \approx \sup _{t>0} t^{-\frac{\lambda}{q}}\left\|s^{\frac{1}{p}-\frac{1}{q}} f^{*}(s)\right\|_{L_{q}(0, t)} \\
& =\|f\|_{M_{p, q ;}^{\text {loc }}} .
\end{aligned}
$$

\section{Boundedness of the maximal operator in the local Morrey-Lorentz spaces}

In this section, the boundedness of the maximal operator $M$ in local Morrey-Lorentz spaces $M_{p, q ; \lambda}^{\text {loc }}\left(\mathbb{R}^{n}\right)$ is proved.

Theorem 2 Let $0<p<\infty, 1 \leq q \leq \infty, 0<\lambda \leq 1$ or $1<p<\infty, 1 \leq q \leq \infty, \lambda=0$. Then the maximal operator $M$ is bounded on the local Morrey-Lorentz spaces $M_{p, q ; \lambda}^{\text {loc }}\left(\mathbb{R}^{n}\right)$. 
Proof By the definition of a norm in local Morrey-Lorentz spaces,

$$
\begin{aligned}
\|M f\|_{M_{p, q ; \lambda}^{\text {loc }}} & =\sup _{r>0} r^{-\frac{\lambda}{q}}\left\|t^{\frac{1}{p}-\frac{1}{q}}(M f)^{*}(t)\right\|_{L_{q}(0, r)} \\
& \leq C \sup _{r>0} r^{-\frac{\lambda}{q}}\left\|t^{\frac{1}{p}-\frac{1}{q}} f^{* *}(t)\right\|_{L_{q}(0, r)} \\
& =C \sup _{r>0} r^{-\frac{\lambda}{q}}\left\|t^{\frac{1}{p}-\frac{1}{q}-1} \int_{0}^{t} f^{*}(s) d s\right\|_{L_{q}(0, r)} .
\end{aligned}
$$

Having applied the generalized Minkowski inequality, we get

$$
\begin{aligned}
\|M f\|_{M_{p, q ; \lambda}^{\mathrm{loc}}} & \leq C \sup _{r>0} r^{-\frac{\lambda}{q}}\left\|t^{\frac{1}{p}-\frac{1}{q}-1} \int_{0}^{t} f^{*}(s) d s\right\|_{L_{q}(0, r)} \\
& =C \sup _{r>0} r^{-\frac{\lambda}{q}}\left(\int_{0}^{r}\left(\int_{0}^{t} f^{*}(s) d s\right)^{q} t^{\frac{q}{p}-1-q} d t\right)^{\frac{1}{q}} \\
& =C \sup _{r>0} r^{-\frac{\lambda}{q}}\left(\int_{0}^{r}\left(\int_{0}^{1} f^{*}(t s) d s\right)^{q} t^{\frac{q}{p}-1} d t\right)^{\frac{1}{q}} \\
& \leq C \sup _{r>0} r^{-\frac{\lambda}{q}} \int_{0}^{1}\left(\int_{0}^{r} f^{*}(t s)^{q} t^{\frac{q}{p}-1} d t\right)^{\frac{1}{q}} d s \\
& \leq C \int_{0}^{1}\left(\sup _{r>0} r^{-\lambda} \int_{0}^{r} f^{*}(t s)^{q} t^{\frac{q}{p}-1} d t\right)^{\frac{1}{q}} d s \\
& =C \int_{0}^{1}\left(\sup _{r>0} r^{-\lambda} \int_{0}^{s r} f^{*}(t)^{q} t^{\frac{q}{p}-1} d t\right)^{\frac{1}{q}} s^{-\frac{1}{p}} d s \\
& =C \int_{0}^{1}\left(\sup _{r>0} r^{-\lambda} \int_{0}^{r} f^{*}(t)^{q} t^{\frac{q}{p}-1} d t\right)^{\frac{1}{q}} s^{-\frac{1}{p}+\frac{\lambda}{q}} d s \\
& =\|f\|_{M_{p, q ; i}^{\mathrm{loc}}} C \int_{0}^{1} s^{-\frac{1}{p}+\frac{\lambda}{q}} d s \\
& =C\left(\frac{1}{p^{\prime}}+\frac{\lambda}{q}\right)^{-1}\|f\|_{M_{p, q ;}^{\mathrm{loc}}}
\end{aligned}
$$

since $-\frac{1}{p}+\frac{\lambda}{q}>-1$.

Therefore, the maximal operator $M$ is bounded on $M_{p, q ; \lambda}^{\text {loc }}\left(\mathbb{R}^{n}\right)$.

Corollary 3 Let $1 \leq p<\infty, 0<\lambda \leq 1$. Then the maximal operator $M$ is bounded in $M_{p, \lambda}^{\text {loc }}\left(\mathbb{R}^{n}\right)$.

Corollary 4 Let $1<p<\infty, 1 \leq q \leq \infty$. Then the maximal operator $M$ is bounded in $L_{p, q}\left(\mathbb{R}^{n}\right)$.

Corollary 5 [29] Let $1<q<\infty$. Then the maximal operator $M$ is bounded in $W L_{q}\left(\mathbb{R}^{n}\right)$. 


\section{Authors' contributions}

This work was carried out in collaboration between all authors. VSG raised these interesting problems in the research. VSG, CA and AS proved the theorems, interpreted the results and wrote the article. All authors defined the research theme, read and approved the manuscript.

\section{Author details}

'Department of Mathematics, Ankara University, Ankara, Turkey. ${ }^{2}$ Department of Mathematics, Ahi Evran University, Kirsehir, Turkey. ${ }^{3}$ Institute of Mathematics and Mechanics of NAS of Azerbaijan, Baku, Azerbaijan.

\section{Acknowledgements}

C Aykol was partially supported by the Turkish Scientific and Technological Research Council (TUBITAK, programme 2211). The research of VS Guliyev was supported by the grant of Ahi Evran University Scientific Research Projects (PYO.FEN.4001.12.18)

\section{Received: 21 April 2013 Accepted: 10 July 2013 Published: 26 July 2013}

\section{References}

1. Lorentz, GG: Some new function spaces. Ann. Math. 51, 37-55 (1950)

2. Lorentz, GG: On the theory of spaces $\Lambda$. Pac. J. Math. 1, 411-429 (1951)

3. Calderón, AP: Intermediate spaces and interpolation, the complex method. Stud. Math. 24, 113-190 (1964)

4. Peetre, J: Nouvelles proprietes d'espaces d'interpolation. C. R. Math. Acad. Sci. Paris 256, 1424-1426 (1963)

5. Lions, JL, Peetre, J: Sur une classe d'espaces d'interpolation. Publ. Math. Inst. Hautes Études Sci. 19, 5-68 (1964)

6. Riviere, NM, Sagher, Y: Interpolation operator between $L^{\infty}$ and $H^{1}$ the real method. J. Funct. Anal. 14, 401-409 (1973)

7. Bennett, C, Sharpley, R: Interpolation of Operators. Academic Press, Boston (1988)

8. Mingione, G: Gradient estimates below the duality exponent. Math. Ann. 346, 571-627 (2010)

9. Adams, DR: Lectures on $L_{p}$-potential theory. Department of Mathematics, University of Umea (1981)

10. Adams, DR, Hedberg, LI: Function Spaces and Potential Theory. Grundlehren der Mathematischen Wissenschaften, vol. 314. Springer, Berlin (1996)

11. Adams, DR, Lewis, JL: On Morrey-Besov inequalities. Stud. Math. 74, 169-182 (1982)

12. Ragusa, MA: Embeddings for Morrey-Lorentz spaces. J. Optim. Theory Appl. 154(2), 491-499 (2012)

13. Guliyev, VS: Integral operators on function spaces on the homogeneous groups and on domains in $\mathbb{R}^{n}$. Doctoral degree dissertation, Mat. Inst. Steklov, Moscow, 1-329 (1994) (Russian)

14. Guliyev, VS: Function spaces, integral operators and two weighted inequalities on homogeneous groups. Some applications. Baku (1999) (Russian)

15. Guliyev, VS, Mustafayev, RC: Integral operators of potential type in spaces of homogeneous type. Dokl. Akad. Nauk, Ross. Akad. Nauk 354(6), 730-732 (1997) (Russian)

16. Guliyev, VS, Mustafayev, RC: Fractional integrals in spaces of functions defined on spaces of homogeneous type. Anal. Math. 24(3), 181-200 (1998) (Russian)

17. Burenkov, Vl, Guliyev, HV: Necessary and sufficient conditions for boundedness of the maximal operator in the local Morrey-type spaces. Stud. Math. 163, 157-176 (2004)

18. Burenkov, VI, Guliyev, HV, Guliyev, VS: Necessary and sufficient conditions for boundedness of fractional maximal operators in local Morrey-type spaces. J. Comput. Appl. Math. 208, 280-301 (2007)

19. Burenkov, Vl, Guliyev, HV, Tararykova, TV, Serbetci, A: Necessary and sufficient conditions for the boundedness of genuine singular integral operators in local Morrey-type spaces. Eurasian Math. J. 1(1), 32-53 (2010)

20. Burenkov, VI, Guliyev, VS: Necessary and sufficient conditions for the boundedness of the Riesz potential in local Morrey-type spaces. Potential Anal. 30, 211-249 (2009)

21. Burenkov, VI, Gogatishvili, A, Guliyev, VS, Mustafayev, RC: Boundedness of the fractional maximal operator in Morrey-type spaces. Complex Var. Elliptic Equ. 55, 739-758 (2010)

22. Komori-Furuya, Y, Matsuoka, K, Nakai, E, Sawano, Y: Integral operators on $B_{\sigma}$-Morrey-Campanato spaces. Rev. Mat. Complut. 26(1), 1-32 (2013)

23. Komori-Furuya, Y, Matsuoka, K, Nakai, E, Sawano, Y: Applications of Littlewood-Paley theory for $B_{\sigma}$-Morrey spaces to the boundedness of integral operators. J. Funct. Spaces Appl. 2013, Article ID 859402 (2013)

24. Morrey, CB: On the solutions of quasi-linear elliptic partial differential equations. Trans. Am. Math. Soc. 43, 126-166 (1938)

25. Peetre, J: On the theory of $\mathcal{L}_{p, \lambda}$ spaces. J. Funct. Anal. 4, 71-87 (1969)

26. Stein, EM, Weiss, G: Introduction to Fourier Analysis on Euclidean Spaces. Princeton University Press, Princeton (1971)

27. Hunt, R: On L(p,q) spaces. Enseign. Math. 12, 249-276 (1966)

28. Grafakos, L: Classical and Modern Fourier Analysis. Pearson Education, Upper Saddle River (2004)

29. Stein, EM: Singular Integrals and Differentiability Properties of Functions. Princeton Math. Ser., vol. 30. Princeton University Press, Princeton (1971)

doi:10.1186/1029-242X-2013-346

Cite this article as: Aykol et al.: Boundedness of the maximal operator in the local Morrey-Lorentz spaces. Journal of Inequalities and Applications 2013 2013:346. 